Revue québécoise de linguistique

\title{
Grammaires en contact : définition et perspectives de recherche
}

\section{Claire Lefebvre}

Volume 14, numéro 1, 1984

Grammaires en contact

URI : https://id.erudit.org/iderudit/602526ar

DOI : https://doi.org/10.7202/602526ar

Aller au sommaire du numéro

Éditeur(s)

Université du Québec à Montréal

ISSN

0710-0167 (imprimé)

1705-4591 (numérique)

Découvrir la revue

Citer cet article

Lefebvre, C. (1984). Grammaires en contact : définition et perspectives de recherche. Revue québécoise de linguistique, 14(1), 11-47.

https://doi.org/10.7202/602526ar d'utilisation que vous pouvez consulter en ligne.

https://apropos.erudit.org/fr/usagers/politique-dutilisation/ 


\title{
GRAMMAIRES EN CONTACT DÉFINITION ET PERSPECTIVES DE RECHERCHE*
}

\author{
Claire Lefebvre
}

\section{Introduction}

Les différentes traditions de recherche sur les langues en contact, qu'elles portent sur les aspects psychologiques (e.g. attitudes, acquisition de langues secondes), ethnographiques (e.g. choix et fonction des codes) ou linguistiques (emprunt, interférence, 'language death', etc.) partagent la caractéristique commune d'avoir traité les phénomènes de contact entre les langues sans toujours départager les facteurs externes des facteurs internes dans la dynamique de l'emprunt linguistique.

L'objet de cet article a trait aux facteurs internes. La problématique adoptée est celle des grammaires en contact plutôt que celle des langues en contact. La notion de 'grammaire' sous-jacente à l'approche adoptée dans cet article correspond à celle des générativistes; elle est telle que définie dans Chomsky (e.g. 1979, 1981) et les travaux qui y sont reliés.

L'article vise à faire ressortir la contribution d'une théorie de la grammaire à la formulation des questions de recherche sur l'emprunt linguistique et les prédictions d'une telle théorie sur les types d'emprunts possibles et impossibles, et sur la nature des changements qui peuvent en

* Cet article est une version élargie de deux communications présentées sous les titres suivants :

«Contraints on borrowing» (University of Toronto, OISE, avril 1983) et "Grammars in contact : issues and perspectives» (University of Buffalo, Symposium on bilingualism, octobre 1983). Je remercie Franco d'Introno, Pieter Muysken, Rajendra Singh, les étudiants du séminaire sur les grammaires en contact UQAM, hiver 1983), Judith McAnulty et Joseph Emonds pour leur discussion fructueuse et leurs précieux commentaires sur les divers points exposés dans cet article. 
découler. À l'intérieur de ce cadre général, l'article s'adresse plus spécifiquement à la question suivante : dans quel(s) composant(s) de la grammaire l'emprunt prend-il place?

La littérature sur l'emprunt suggère que l'emprunt linguistique prend place dans tous les composants de la grammaire. L'emprunt lexical est le plus répandu et le mieux documenté; la lecture des ouvrages sur l'emprunt lexical suggère que tous les types d'items lexicaux peuvent être empruntés. L'emprunt de morphèmes liés est considéré comme possible mais peu fréquent. Cependant, plusieurs cas de morphèmes dont l'origine peut être retracée dans une autre langue que la langue cible sont rapportés dans la littérature. Enfin, une littérature abondante fait état de cas d'emprunts syntaxiques, phénomènes mieux connus sous le nom de «convergence linguistique» (Weinreich 1953).

Les conclusions majeures qui découlent des analyses proposées dans cet article sont les suivantes :

1) Le composant lexical se présente comme le composant privilégié de l'emprunt. Les types d'items pouvant être empruntés sont limités entre autres par les configurations de traits syntaxiques disponibles dans la langue cible. L'emprunt lexical peut donner lieu à des cas de réanalyses morphologiques (et certainement phonologiques, un point qui n'est pas traité dans cet article). L'emprunt syntaxique est virtuellement impossible. Pour qu'il se produise il faut que la langue source contienne une structure qui soit compatible avec les changements internes possibles dans la langue cible, ce qui fait reposer l'emprunt éventuel sur la situation interne de la langue cible. Cette conclusion a pour conséquence de rendre les cas de convergence syntaxique virtuellement impossibles; en cela les conclusions des analyses présentées dans cet article s'opposent directement à celles de Gumperz et Wilson (1971).

2) Les types d'emprunts possibles découlent d'une théorie de la grammaire de sorte qu'il n'est pas nécessaire d'avoir recours à une théorie auxiliaire pour rendre compte de l'emprunt linguistique.

3) Les types de changements qui découlent de l'emprunt sont très limités et ils sont de même nature que les changements internes qui ne découlent pas de l'emprunt, ce qui renforce la théorie selon laquelle le changement ne peut être qu'interne (v.g. Lightfoot 1979). 
4) Si une théorie adéquate de la grammaire prédit les types d'emprunts possibles et les changements qui peuvent en découler, les données d'emprunt linguistique sont utilisables dans l'élaboration de théories linguistiques; cette conclusion est contraire à la position de Chomsky selon laquelle «each actual language will incorporate a large periphery of borrowings, historical residues, inventions and so on, that we can hardly expect to, and indeed would not want to, incorporate within a theory of universal grammar». (Chomsky 1981, p. 8).

Une remarque méthodologique s'impose à ce point. Les analyses proposées dans cet article sont basées sur des données provenant en majeure partie du contact entre l'espagnol et le quechua de la région andine et du créole haïtien, langue de contact par définition ${ }^{1}$. Les conclusions théoriques générales qui se dégagent de ces analyses doivent, si elles sont justes, être valides pour des données similaires provenant d'autres langues sans qu'il ne soit nécessaire de réanalyser les données de ces autres langues qui ont mené les chercheurs à des conclusions différentes des nôtres. Chacun doit faire valoir ses arguments et les conclusions qu'il en tire à partir de données qu'il connaît.

\section{L'article est organisé de la façon suivante :}

La section 2 porte sur l'emprunt lexical (le cas clair), la section 3 sur l'emprunt morphologique (le cas intermédiaire) et la section 4 sur l'emprunt syntaxique (le cas virtuellement impossible). Pour chacun de ces points, une brève revue de la littérature est présentée, des questions de recherche sont formulées dans le cadre de la théorie de Chomsky (1981) et des travaux qui y sont reliés (v.g. Aronoff 1976, Jackendoff 1977). Dans chacune des sections, des cas types d'emprunt sont analysés en regard des questions de recherche proposées. La section 5 contient une discussion sur les conclusions des analyses proposées et sur les perspectives de recherche qu'offre l'étude des grammaires en contact tant pour l'étude des phénomènes de contact entre les langues que pour la théorie linguistique.

2. L'emprunt lexical

\subsection{Revue de la littérature}

D'un survol de la littérature classique sur l'emprunt lexical (Whitney 1881, Meillet 1921, Sapir 1927, Schuchardt 1928, Tresnière 1939, Haugen

1. À moins qu'il ne soit mentionné autrement, les données analysées dans cet article sont des données originales que $\mathrm{j}$ 'ai recueillies sur le terrain au cours des quinze dernières années, à l'occasion de mon travail sur la langue quechua et sur l'haïtien (voir bibliogaphie). 
1950, Weinreich 1953), on peut tirer deux conclusions : 1) tous les types d'items lexicaux peuvent être empruntés, et 2) les emprunts à incidence syntaxique, c'est-à-dire ceux impliquant la morphologie flexionnelle ou des catégories lexicales mineures comme les auxiliaires, sont plus rares que ceux qui ne comportent pas de contenu syntaxique. Les facteurs linguistiques qui ont été proposés comme contraintes sur l'emprunt lexical sont les suivants :

(i) la fréquence d'utilisation des mots : «Frequent words come easily to mind and are therefore more stable.» (Weinreich 1953, p. 57);

(ii) le vocabulaire de base : le vocabulaire de base établi par Swadesh (1952, p. 452) et dont il a été démontré qu'il changeait à un rythme moins rapide que le vocabulaire dit culturel, est moins perméable à l'emprunt (Hymes 1971);

(iii) la congruence des structures des langues en contact;

(iv) la catégorie syntaxique des items lexicaux : cette contrainte est exprimée sous forme de hiérarchies définissant la probabilité relative d'empruntabilité pour chaque catégorie lexicale. Voici quelques exemples de hiérarchies proposées (tiré de Muysken 1980) :

Whitney (1881) N - other parts of speech — suffixes - inflection sounds

Haugen (1950) $\mathrm{N}-\mathrm{V}-\mathrm{A}-\mathrm{ADV}-\mathrm{P}$ Interjections

Singh (1981) $\quad N-A-V-P$

Muysken (1981) N - A - V - P - Conj. - DET - Pron Clit. - COMP

Ces contraintes, toutes probabilistes, reposent sur la présomption que tous les types d'emprunts sont possibles. Elles ne spécifient pas les processus impliqués dans l'emprunt lexical ni les contraintes imposées sur ces processus pour chaque type d'emprunt lexical. Tel que souligné par Weinreich (1953), la contrainte (iii) n'est pas vérifiée par les faits. Les cas de relexification discutés plus bas impliquant des langues de structures complètements différentes (e.g. l'haïtien (Lefebvre 1982)) la media lengua (Muysken 1980) constituent des contre-exemples éclatants à la contrainte (iii). Que peut-on apprendre de plus au sujet des contraintes sur l'emprunt lexical si on étudie l'emprunt lexical dans le cadre d'une théorie formelle de la grammaire, et si l'on considère les types d'emprunt relativement aux processus d'emprunt disponibles. 
Avant de passer à la formulation de questions de recherche pour l'étude de l'emprunt lexical dans le cadre d'une théorie de la grammaire, une définition sommaire des processus d'emprunt s'impose. Ces processus incluent la relexification, la lexification et la translexification. La lexification consiste en un emprunt lexical pour fournir une matrice phonétique à une catégorie syntaxique déjà existante dans la langue cible mais pour laquelle il n'y a pas d'item lexical disponible dans la langue. Un exemple de lexification serait l'emprunt d'un item lexical à une autre langue pour fournir une matrice phonétique à la catégorie 'complémenteur' de la langue cible, dans laquelle il n'y a pas d'item lexical complémenteur. La relexification est un processus par lequel un item lexical emprunté remplace un item lexical original tout en conservant la matrice de traits syntaxiques de l'item lexical qu'il remplace dans la langue cible. Ce processus peut être représenté graphiquement de la façon suivante :

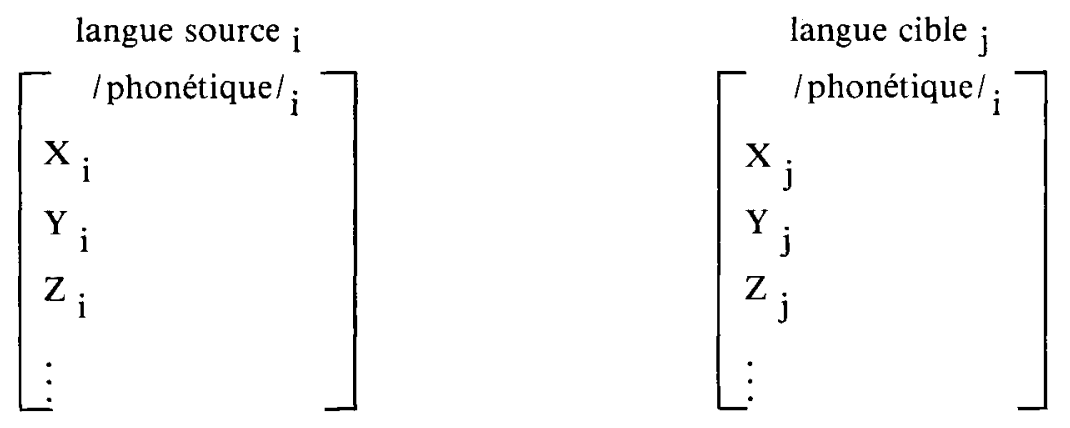

La translexification est le processus par lequel l'item lexical emprunté est introduit dans la langue cible avec certains des traits syntaxiques qu'il a dans la langue source :

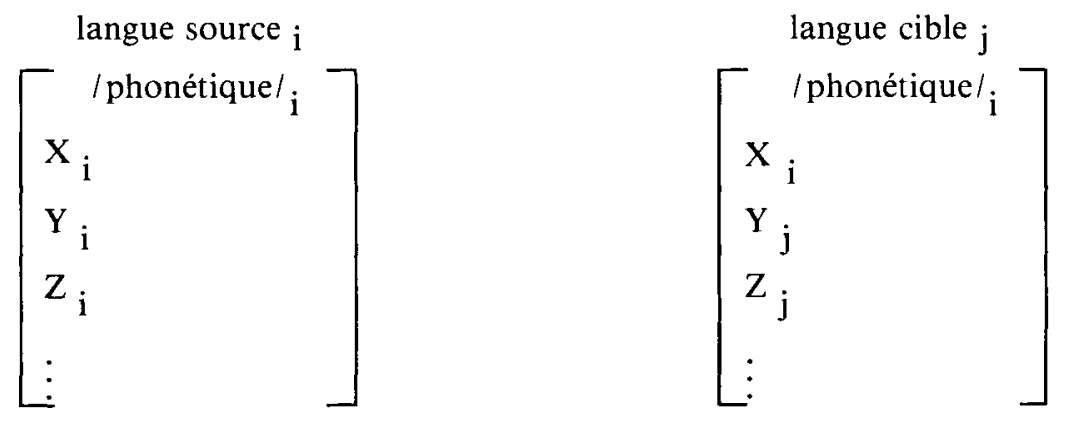

Les autres processus d'emprunt, plus spécifiques, seront définis au fur et à mesure de la discussion. 


\subsection{Questions de recherche}

$\mathrm{Si}$ on prend pour acquis une théorie de la base contenant les règles de base, qui ont le format général de la théorie X' (Jackendoff 1977) et un lexique, les règles de base et le lexique étant reliés à travers les traits et les catégories syntaxiques, nous pouvons formuler les questions de recherche suivantes relativement aux contraintes sur l'emprunt lexical :

1) La catégorie syntaxique des items lexicaux : est-il possible d'emprunter des mots définis par des traits syntaxiques mineurs (v.g. [ \pm temps], DET, etc.) aussi bien que des mots définis par des traits syntaxiques majeurs (v.g. $\pm \mathrm{N}, \pm \mathrm{V})$ ? Par exemple, est-il possible d'emprunter des complémenteurs, des auxiliaires, des déterminants, etc., à une autre langue, aussi bien que des noms, des verbes, des adjectifs et des prépositions? Dans quels processus d'emprunt ces éléments peuvent-ils être impliqués : lexification, translexification, relexification, etc.?

2) Les catégories syntaxiques disponibles dans la langue cible: est-ce qu'une langue peut emprunter comme tel un mot d'une catégorie lexicale qu'elle n'a pas? Par exemple, une langue qui n'a pas la catégorie lexicale $\mathbf{P}$ (préposition) peut-elle emprunter des prépositions à une autre langue? Cette possibilité aurait comme conséquence qu'une catégorie syntaxique nouvelle pourrait être créée directement par l'emprunt lexical, affectant ainsi la structure du lexique et des règles de base de la langue emprunteuse.

3) Les facteurs paradigmatiques : une langue peut-elle emprunter des items lexicaux qui devraient être incorporés dans un paradigme déjà structuré? Par exemple, une langue peut-elle emprunter des conjonctions, des mots Wh, des clitiques, etc.?

4) Les facteurs syntagmatiques : les éléments relevant de la grammaire du discours sont-ils plus perméables à l'emprunt que ceux relevant de la grammaire de la phrase? Par exemple, observe-t-on une différence dans l'emprunt de conjonctions selon qu'elles relèvent de la syntaxe de la phrase ou de celle du discours?

5) Quels sont les processus impliqués dans l'emprunt lexical (relexification, translexification, réanalyse interne, etc.) et à quelles catégories syntaxiques ces processus s'appliquent-ils? $\mathrm{Y}$ a-t-il des contraintes sur les processus impliqués dans l'emprunt relativement au type d'items lexicaux empruntés? 
6) Quel(s) type(s) de changement l'emprunt peut-il entraîner?

Dans la section 2.3, ces différentes questions sont explorées à travers l'étude de quelques cas d'emprunt; en 2.4 , ces questions sont discutées à la lumière des cas analysés.

\section{3 Études de cas}

\subsubsection{L'emprunt à l'espagnol de si et sièske en quechua de Cochabamba}

Le quechua est une langue SOV avec un COMP final. La subordination y est encodée de deux manières. Dans le premier cas le verbe contient un affixe de subordination occupant la même position dans le mot que les suffixes de temps dans les phrases à temps fini, et la phrase enchâssée ne contient pas de complémenteur lexical. Dans le second cas le verbe contient un affixe de temps et la phrase enchâssée contient obligatoirement un complémenteur lexical. Dans les deux cas, la phrase subordonnée occupe la position TOPIC de la phrase matrice ou elle est adjointe à $S$ (voir Lefebvre 1980). Dans aucun cas la subordonnée n'est enchâssée. Les phrases (1) et (2) illustrent ces deux possibilités.

(1) [ [warmi riku-qti-y-mi] rima-wa-nqa]

S S'

femme voir SUB 1 VAL parler $13 F U$

(Cuzco et autres dialectes quechuas)

Si je vois la femme elle me parlera

(2) [warmi-ta riku-ni cayqa] [pay-mi rima-wa-nqa]

Top $S^{\prime}$

femme AC voir 1 COMP elle VAL parler $13 \mathrm{FU}$ (Cuzco et autres (dialectes quechuas)

Si je la vois la femme elle me parlera

En plus de ces deux constructions, on trouve, en quechua de Cochabamba (et de Cuzco), des subordonnées équivalentes à (1) et (2), introduites par

2. Si a également été emprunté dans d'autres dialectes du quechua. Pour les fins de cette analyse, j'ai choisi le dialecte de Cochabamba pour plusieurs raisons : 1) il est bien documenté; 2) il est moins conservateur que plusieurs des autres dialectes et 3) les changements entourant l'emprunt de si offrent plus d'ampleur pour les fins de cette discussion. 
si/syèske, empruntés à l'espagnol : si 'si'/sièsque 's'il est que' (Lastra 1968, Bills et al. 1972), tel qu'illustré en (3) et (4) respectivement.

(3) si-cus suyaykuma-nqa, wagay-n-man

si attendre 3FU appeler 3 POT

S'il attend, il l'appellera

(Cochabamba, Bills et $a l$,

(4) syèske-cus qan ka-y-man, muc'a-y-man

si toi être 1 POT embrasser 1 POT

1971, p. 30)

(Cochabamba,

Bills et al.,

Si j'étais toi, je l'embrasserais

1972, p. 30)

Dans ces phrases, le verbe est tensé, comme dans la subordonnée de (2). Si apparaît au début de la phrase subordonnée plutôt qu'à la fin, comme c'est le cas en (2). L'introduction de si a-t-elle créé une position syntaxique de COMP initial dans une langue où le COMP est final? Je montrerai que tel n'est pas le cas en argumentant que si a pu être emprunté et occupe cette position parce que la langue cible avait déjà développé de façon interne une position de COMP initial rempli par -cus (suffixé à si dans les exemples (3) et (4)). Dans un premier temps, je montrerai que -cus, suffixe validationnel, a été réinterprété comme complémenteur initial. Dans un deuxième temps, j'explorerai les conséquences de cette réanalyse interne pour le quechua de Cochabamba et les possibilités d'emprunt lexical à l'espagnol.

Le quechua possède une classe de suffixes validationnels, représentés en (5).

(5) $-\min /-n:$ affirmatif

-si/-s : ouie-dire

-ca : dubitatif

-cu : interrogatif

(6) a. $\mathrm{ka}-\mathrm{n}-\mathrm{mi}$

être $3 \mathrm{AF}$

Il y en a (affirmatif)

b. $\mathrm{ka}-\mathrm{n}-\mathrm{si}$

être 3 ouie-dire

Il y en a (j'ai entendu dire)

Les propriétés de ces suffixes qui sont pertinentes pour la présente analyse sont présentées en (7) : (Pour plus de détails, voir Muysken 1979 et Lefebvre et Muysken (à paraître)). 
— ils sont indépendants, c'est-à-dire qu'ils ne sont pas engendrés par des règles de formation de mot;

- ils n'apparaissent que dans les phrases matrices;

- leur champ porte sur toute la phrase dans laquelle ils se trouvent quel que soit le constituant auquel ils sont attachés;

- il ne peut y en avoir qu'un par proposition.

Lefebvre et Muysken (à paraître) argumentent en faveur d'une analyse selon laquelle ces suffixes sont engendrés sous $S$ par une règle de base de type (8).

$\mathrm{S} \rightarrow$ VAL....NP....(NEG)....VP....INFL

Les propriétés des validateurs listées en (7) sont incompatibles avec une analyse de ces suffixes comme complémenteurs. En effet, le seul fait qu'ils n'apparaissent que dans les racines exclut une telle hypothèse (voir Lefebvre et Muysken 1978).

Quel est le statut du suffixe-cus apparaissant dans les phrases (3) et (4)? Bills et al.(1972) et Lastra (1968) considèrent -cus comme un suffixe indépendant dubitatif appartenant à la classe des validateurs. Certains des exemples qu'ils fournissent pour appuyer cette analyse, dont (9) et (10), montrent que -cus partage en effet les propriétés ci-haut décrites des validateurs.

$$
\begin{aligned}
& \text { hina - cus, mana - cus } \\
& \text { ainsi VAL non VAL }
\end{aligned}
$$

Est-ce ainsi ou n'est-ce pas ainsi?

(Bills et al., 1972, p. 165)

(10) hamu - nqa-cus mana - cus, mana yaca - ni - cu venir 3FU VAL non VAL non savoir 1 NEG

Je ne sais pas s'il viendra ou non

(Bills et al., 1972, p. 165)

Bills et al. fournissent cependant certaines données où -cus ne présente pas les propriétés des validateurs. Par exemple, dans les exemples (3) et (4), -cus apparaît à l'intérieur de la phrase subordonnée. L'analyse qui suit est ma réanalyse de leurs données, ainsi que de données recueillies auprès d'un informateur cochabambaien ${ }^{3}$. Premièrement, on trouve -cus dans les phrases subordonnées telles que (11) et (12), ce qui constitue une exception au fait que les validateurs n'apparaissent que dans les phrases matrices.

3. Je remercie Basilio Medrano, originaire de Cochabamba, pour sa contribution à la présente analyse en sa qualité d'informateur 
(11) má yaca - ni - cu ima - ta -cus ruwa - saq QUESTION INDIRECTE PAS savoir 1 NEG quoi AC VAL faire 1FU

Je ne sais pas ce que je vais faire

(Bills et al., 1972, p. 273)

(12) rik $^{h_{u}}$ - ni warmi - ta pi - ta -cus felipe muc'a - rqa

RELATIVE voir 1 femme AC qui AC VAL Philippe embrasser PA

J'ai vu la femme que Philippe a embrassée.

(Bills et al., 1972, p. 273)

Deuxièmement, dans ces phrases, -cus n'a pas le sens dubitatif-conditionnel qu'il a dans les phrases matrices (9) et (10); ceci est particulièrement évident en (12) où -cus semble être vide de sens. Dans cette phrase, -cus n'est donc plus analysable comme validateur. Je propose que, dans cet environnement, -cus soit la réalisation morphologique du trait $\mathrm{T}$ (emps) dans COMP. Le fait que -cus soit en distribution complémentaire avec les suffixes de subordination sur le verbe supporte cette analyse. Comparez les phrases de (13).

(13) a. qulqi - y ka - qti - n - qa

argent 1 être SUB 3 TOPIC

Si j'avais de l'argent

b. ${ }^{*}$ qu1qi - y-cus ka - qti - n - qa

argent 1 COMP être SUB 3 TOPIC

Si j'avais de l'argent

c. qulqi - y -cus ka - n - man

argent 1 COMP être 3 POT

Si j'avais de l'argent

En (13a), la subordination est encodée par le suffixe de subordination (SUB) affixé au verbe. (13b), où -cus apparaît dans la même phrase que le subordonnant -qti, n'est pas grammaticale. En (13c), on trouve -cus, un verbe à temps fini (Potentiel) et il n'y a pas de complémenteur final. Dans les phrases de (13), -cus est donc en distribution complémentaire avec un suffixe de subordination sur le verbe et avec un complémenteur lexical final. La distribution milite donc dans ce contexte en faveur d'une analyse de -cus comme complémenteur.

(14) a. qulqi - y - cus ka - n - man cayqa argent 1 Val? être 3 POT COMP

Si j'avais de l'argent 
Cette phrase est citée par Lastra $(1968, p .62)$ et elle est acceptée comme grammaticale par notre informateur cochabambaien. Est-elle un contreexemple à l'analyse de -cus comme complémenteur? Si -cus y est interprété comme complémenteur, la phrase (14) contient alors deux complémenteurs : -cus et cayqa. Pour notre informateur, -cus, en (14 a), n'est pas interprétable comme la réalisation de COMP mais comme un validateur. En effet, pour lui, il y a une différence de sens entre (14 a) et (14 b) relative à la présence et à l'absence de -cus respectivement.

(14) b.qulqi - y ka - n - man cayqa

argent 1 être 3 POT COMP

Si j'avais de l'argent

(14a) ne peut être interprétée qu'avec un sens dubitatif (e.g. si j'avais de l'argent, mais cette possibilité est improbable), alors que (14b) a un sens plus affirmatif (e.g. si j'avais de l'argent/quand j'aurai de l'argent). Cette distinction correspond au sens de -cus comme validateur et non comme complémenteur. La grammaticalité de (14a) suggère donc que la réanalyse du validateur -cus en complémenteur est passée par un stade où -cus (dubitatif) a été utilisé comme suffixe indépendant dans les phrases enchâssées tensées. En acquérant le statut de complémenteur, il perd son sens initial dubitatif-conditionnel, tel qu'illustré en (11), (12) et (13).

Un autre ensemble de faits confirme l'analyse proposée. En qualité de validateur (donc engendré sous S), -cus ne peut apparaître qu'avec le mode conditionnel, tel qu'illustré par le contraste de grammaticalité entre les phrases de (15).

(15) a. qulqi - y - cus ka - n - man cayqa argent 1 VAL être 3 POT COMP

Si j'avais de l'argent

b. ${ }^{*}$ qulqi - y - cus ka - nqa cayqa argent 1 VAL être 3FU COMP

Si j'avais de l'argent

c. qulqi - y ka - nqa cayqa argent 1 être 3FU COMP

Si j'avais de l'argent

En effet, en (15 a) -cus apparaît avec un verbe au conditionnel (POT). Alors que $(15 \mathrm{c})$ où -cus n'apparaît pas et où le verbe est au futur est grammaticale, ( $15 \mathrm{~b})$ où -cus apparaît et où le verbe est au futur n'est pas 
grammaticale. Comme complémenteur, -cus peut donc apparaître avec un verbe à temps fini quel qu'il soit; ainsi (11) contient un verbe au futur et (12) contient un verbe au passé.

Si notre analyse est juste, le quechua de Cochabamba a développé un complémenteur initial par un processus de réanalyse interne d'un validateur en complémenteur. La règle de réécriture de $S$ ' en quechua de Cochabamba inclut donc un COMP initial ou un COMP final, tel que représenté en (15) ${ }^{4}$.

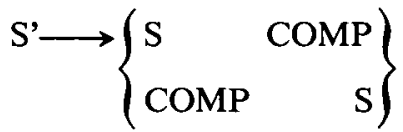

Quelles sont les conséquences de la création d'un COMP initial en quechua? Premièrement, la création d'un COMP initial permet l'enchâssement de phrases subordonnées contenant un verbe tensé et un complémenteur initial, tel qu'illustré en (11) et (12). Ceci représente une innovation dans la langue, les phrases subordonnées du type de celles analysées ici occupant la position topique ou étant adjointes à $\mathrm{S}$, tel que mentionné précédemment. Ce cas sera discuté plus en détail dans la section 4 sur l'emprunt syntaxique.

Deuxièmement, ce changement interne a permis l'emprunt récent de si/sièske pour introduire des phrases subordonnées conditionnelles, telles qu'en (3) et (4). En effet, en acquérant le statut de complémenteur, -cus a perdu son sens initial de marqueur dubitatif; le quechua ne possède pas d'item lexical équivalent à $s i$; à la suite de la réanalyse de -cus en complémenteur, il fallait créer un item lexical pour introduire les phrases conditionnelles. Cet item lexical ne pouvait être cayqa, complémenteur final, ce dernier n'étant pas équivalent à si. En effet, les phrases de type $\mathrm{S}$ cayqa peuvent recevoir deux interprétations en quechua: l'une 'tense relative' (terme emprunté à Hale 1975) recevant une interprétation conditionnelle, l'autre 'noun relative', recevant une interprétation relative (voir Lefebvre et Muysken 1982). L'item lexical manquant a donc été emprunté à l'espagnol. Le fait que si et sièske apparaissent avec le complémenteur -cus suggèrent que si et sièske ont été empruntés comme mots Wh et non comme complémenteurs. Ceci est confirmé par le fait que $s i$ et sièske doivent porter le suffixe -cus comme en (3) et (4), à défaut de quoi, le complémenteur final cayqa doit apparaître dans la phrase subordonnée,

4. Notons ici que la structure interne des deux COMP n'est pas la même. Le COMP final contient $\alpha \mathrm{T}$ et CASE (voir Lefebvre 1980 et Lefebvre et Muysken (à paraître)). Le COMP initial contient une position Wh et une position $\alpha \mathrm{T}$. 
tel qu'illustré en (16), citée par Lastra (1968), et acceptée par mon informateur cochabambaien.

(16) si qulqi - y ka - n - man cayqa

si argent 1 être 3 POT COMP

Si j'avais de l'argent

(Lastra 1968, p. 62)

$s i$ avait les propriétés d'un complémenteur plutôt que celles d'un mot Wh, la phrase (16) contiendrait deux complémenteurs.

La cooccurrence de -cus et cayqa avec si n'est cependant pas possible :

$(17) *$ si - cus qulqi - y ka - n - man cayqa

si COMP argent 1 être 3 POT COMP

Si j'avais de l'argent

Cette analyse montre donc que ce n'est pas l'introduction du si de l'espagnol qui a créé la catégorie initiale COMP en quechua de Cochabamba, mais que cette création résulte d'un développement interne dans la langue, lequel a permis l'introduction d'items lexicaux empruntés à l'espagnol pouvant occuper cette position ${ }^{5}$. Nous concluons donc que l'emprunt lexical de si/sièske est venu combler un trou lexical pour une position syntaxique déjà existante dans la langue et que si et sièske ont été introduits dans le paradigme d'une catégorie lexicale déjà existante : celle des mots $\mathrm{Wh}^{6}$.

En généralisant les résultats de cette analyse, on peut faire l'hypothèse que l'emprunt lexical ne peut créer une position syntaxique qui n'existe pas dans la langue emprunteuse.

\subsubsection{La formation de la media lengua : un cas de relexification}

Le second cas considéré est celui de la formation de la media lengua. Les données et l'analyse présentées sont tirées de Muysken (1980). La media lengua est une langue de contact, parlée dans les communautés andines de l'Équateur. Cette langue existe depuis une soixantaine d'années et elle est parlée, soit comme langue maternelle ou comme deuxième ou troisième

5. En plus de rapporter l'emprunt à l'espagnol de si et sieske en quechua de Cochabamba, Lastra (1968, p. 62) donne un exemple contenant porke-cus, 'parce que' et un exemple contenant cuando, 'quand'. Notre informateur atteste l'existence de porke dans son dialecte mais rejette cuando comme emprunt à l'espagnol.

6. Un contre-exemple possible à cette conclusion serait un dialecte du quechua où si aurait été emprunté à l'espagnol sans qu'on ne puisse montrer qu'un élément du type de -cus ait été réanalysé comme complémenteur. À ma connaissance, un tel cas n'existe pas. 
langue. Ce cas de contact linguistique est d'autant plus intéressant qu'il implique deux langues dont les structures ne sont pas congruentes.

En effet, le quechua est une langue SOV avec une morphologie riche; l'espagnol est une langue romane avec un ordre de mots de base SVO ou VSO (selon Bordelois) et une morphologie relativement pauvre comparativement au quechua.

La media lengua présente une structure grammaticale essentiellement quechua et un lexique à $90 \%$ espagnol. La formation de cette langue se caractérise par l'emprunt d'items lexicaux dont les traits syntaxiques sont ceux qui caractérisent les items lexicaux remplacés. Ce processus est connu sous le nom de relexification. L'exemple (1) contraste une phrase quechua et la phrase équivalente en media lengua.

Yalli - da tamia - pi - ga, mana ri - sha - chu
dimas - ta llubi - pi - ga, no i -sha - chu
trop AC pluie SUB TO non aller PR NEG
Si llueve demas, no voy a ir
S'il pleut trop je n'irai pas

QUECHUA

MEDIA LENGUA

trop AC pluie SUB TO non aller PR NEG

S'il pleut trop je n'irai pas

Selon Muysken, la media lengua a préservé la grammaire quechua presque entièrement. En (1), on peut observer un ordre de mots SOV, l'usage des affixes verbaux du quechua, par exemple, le suffixe de subordination - $p i$, le marqueur de topique - $g a$ et le suffixe de négation -cu; les marqueurs de personne et de temps sont affixés aux racines, comme en quechua, et non comme en espagnol. Les éléments relexifiés incluent les noms, les pronoms personnels forts, les verbes, les adjectifs, les conjonctions, les mots Wh et les pronoms déictiques.

Dans quelle mesure les éléments relexifiés correspondent-ils aux catégories lexicales déjà existantes en quechua? Le tableau de (2), tiré de Muysken (1980), présente une liste comparée des catégories lexicales de l'espagnol $(\mathrm{Sp})$, du quechua $(\mathrm{Q})$ et de la media lengua $(\mathrm{ML})$.

(2) $\mathrm{Sp}$

N

A

$\mathrm{P}$

CONJUNCTION
Q

$\mathrm{N}$

V

A

$-?$

$-\mathrm{CONJ}$
ML

$\mathbf{N}$

V

A

(P)

CONJ, -CONJ MINOR 


$\begin{array}{lll}\text { COMPLEMENTIZER } & - & - \\ \text { CLITIC PRONOUN } & - & - \\ \text { WH-PRONOUN } & \text { WH-PRO } & \text { WH-PRO } \\ \text { DEICTIC PRONOUN } & \text { DEI PRO } & \text { DEI PRO }\end{array}$

Ce tableau révèle que seuls les éléments lexicaux appartenant à des catégories syntaxiques déjà existantes dans la langue quechua ont été relexifiés. Quelques exceptions potentielles sont notées par Muysken, par exemple, la présence de certaines prépositions et de conjonctions de l'espagnol. Muysken attribue au 'code switching' la présence de certaines des prépositions espagnoles trouvées dans ses données. Pour ce qui est de la préposition entre 'entre', il écrit : «The instances of /entre/ in ML could be regarded as relexifications of the $\mathrm{Q}$ post-nominal element /-pura/, ... /-pura/ may well be one of the rare instance of a true postposition (P) in Quechua, and hence the relexification as /entre/ would be expected». (pp. 9 et 10). Les cas d'emprunts de conjonctions semblent à première vue plus problématiques. Muysken écrit : "Are the Spanish conjonctions that we find in ML cases of relexification of Quechua categories or cases of introduction from Spanish? The latter option seems the correct one since (a) in Media Lengua the conjunctions are used as in Spanish; (b) they coexist with the Quechua cliticized conjunctions; (c) in Quechua itself we find frequent borrowing of Spanish conjunctions. The only reason probably why we find so few Spanish conjunctions in the CF sample is that only relatively short sentences were eleciteded. Thus the introduction into ML of Spanish conjunctions is the only major exception to the idea that ML lexical categories, arising through relexification correspond strictly to Quechua categories. This exception may be explained by the fact that conjunctions which occur at discourse level are less closely integrated into the grammar of the language and can be borrowed more easily.» (p. 11). Les études de Roy (1979) semblent appuyer cette dernière suggestion de Muysken. Roy note qu'en français de Moncton, on trouve plusieurs conjonctions empruntées à l'anglais (e.g. but 'mais', so 'alors', and, 'et') mais que ces dernières n'apparaissent jamais à l'intérieur d'une phrase entre deux syntagmes; elles n'apparaissent que comme coordonnants de phrases ou comme marqueurs d'interaction. Leur usage est donc exclu de la syntaxe de la phrase et restreint à la syntaxe du discours.

Si cette analyse est correcte, seules les catégories lexicales existantes en quechua et pertinentes à la syntaxe de la phrase ont été relexifiées. 
Quelles conséquences la relexification a-t-elle eu pour la syntaxe de la media lengua? Muysken montre que la relexification a entraîné très peu de changements dans la syntaxe originale. Il note certains changements dans les traits de sous-catégorisation, ainsi que des changements mineurs dans l'ordre des mots. (3) illustre comment un adjectif prénominal en quechua a été relexifié par un adjectif post-nominal en media lengua.

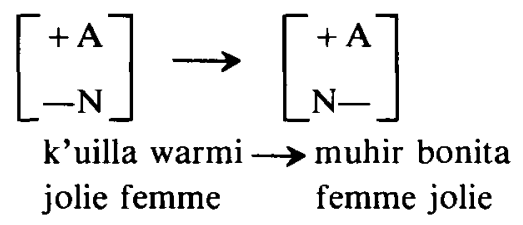

D'autres cas marginaux de changements potentiels sont discutés par l'auteur mais aucun d'eux n'est retenu comme un cas clair de changement en cours dû à la relexification.

Les observations suivantes se dégagent de cette analyse :

1) Même dans le cas d'une relexification massive du lexique d'une langue, l'emprunt ne peut créer de catérogie lexicale dans la langue cible; seuls les éléments lexicaux appartenant à une catégorie lexicale existante dans la langue emprunteuse peuvent être relexifiés.

2) L'emprunt des conjonctions au niveau de la syntaxe du discours mais non au niveau de la syntaxe de la phrase suggère que les contraintes sur l'emprunt lexical doivent être exprimées au niveau de la syntaxe de la phrase, la syntaxe du discours étant plus perméable à l'emprunt que cette dernière.

3) Une troisième constatation se dégage de cette analyse : les changements linguistiques entraînés par la relexification sont mineurs; dans ce cas, ils n'impliquent que des changements dans les traits de souscatégorisation et un changement dans l'ordre des mots à l'intérieur d'un constituant donné.

\subsubsection{La formation du lexique de l'haitien}

Dans Lefebvre (1982), la relexification est proposée comme le processus ayant présidé à la formation de l'haïtien. Il est suggéré que la structure grammaticale correspond en grande partie à celle des langues d'Afrique de l'Ouest (les spécificateurs suivent la tête, le déterminant peut modifier une phrase, la présence dans la langue de verbes sériels, de prédicats clivés, de verbes redoublés; les différents éléments réalisant AUX 
sont encodés par des particules, etc.) alors que le lexique est composé à plus de $90 \%$ d'items lexicaux provenant du français. Une analyse détaillée du processus de relexification en haïtien est actuellement en préparation (voir Kaye et Lefebvre en préparation). Je me contenterai ici d'étudier l'origine des catégories lexicales mineures de cette langue afin de répondre à quelques-unes des questions posées au début de cette section sur l'emprunt lexical, et auxquelles les données présentées jusqu'à maintenant ne permettent pas de répondre.

En haïtien, la grande majorité des particules ou éléments lexicaux mineurs (c'est-à-dire ceux qui réalisent les positions syntaxiques mineures) proviennent d'éléments lexicaux, qui, en français, sont définis par des traits majeurs. Ainsi, les particules réalisant les différents éléments de AUX proviennent de verbes pleins ou d'adverbes français.

TEMPS te [+ antérieur] ' 'été' (participe passé)

MODE a, av, va, ava [-réalisé] '‘va' (verbe tensé) pu [-réalisé] 〈'pouvoir' (verbe) (mode)

ASPECT ap [+ ponctuel] ('après' (adverbe)

Dans tous ces cas, il semble que des éléments lexicaux définis par des traits majeurs aient été empruntés comme tels puis réanalysés par un processus de réanalyse interne, en items lexicaux mineurs pour lexicaliser des positions syntaxiques mineures. Je me limiterai ici à illustrer ce phénomène à partir de l'analyse de la lexicalisation de COMP présentée dans Koopman et Lefebvre (1982).

L'haïtien n'a pas, lors de sa formation, emprunté le complémenteur $q u e$ du français. La lexicalisation de COMP a plutôt été effectuée au moyen de réanalyse à l'intérieur de la langue; ainsi, le complémenteur actuel $p u$ a deux sources : l'une prépositionnelle, provenant de la réanalyse interne du syntagme prépositionnel $\mathrm{PP} \rightarrow \mathrm{P}$ NP en $\mathrm{PP} \rightarrow \mathrm{P}\left\{\mathrm{S}^{\prime}\right\}$, , l'autre modale, provenant de la réanalyse du $p u$, en complémenteur, (voir G. Sankoff et Laberge 1973, G. Sankoff et Brown 1976 et Sankoff 1980 pour ces cas multiples de réanalyse interne de ce type en tok pisin).

Les mots Wh de l'haïtien sont formés sur le modèle des mots Wh de plusieurs langues d'Afrique de l'Ouest : un élément Wh invariant est combiné avec un item nominal référant à 'personne', 'place', 'manière', etc. Pour l'haïtien, l'élément Wh invariant est $k i$. La liste des mots Wh ainsi formés figure en (1) : 
(1) ki - mun (litt. quelle personne) :qui

ki - lès (litt. quelle personne) :qui

ki - kote (litt. quel endroit) :où

$\mathrm{ki}$ - b (litt. quel bord) :où

ki - sa (litt. quelle chose) :quoi

ki - lè (litt. quelle heure) :quand

ki - żã (litt. quel genre) : :comment

Dans ce cas, l'haïtien semble donc avoir relexifié le paradigme des mots Wh des langues d'Afrique de l'Ouest au moyen du procédé morphologique opérant dans ces langues tout en utilisant du matériel lexical français.

Jusqu'à maintenant, nous n'avons vu aucun cas d'emprunts d'items lexicaux mineurs au français dans le processus de relexification. Le fait est que ce phénomène est rare. On n'en trouve que deux cas en haïtien : celui du marqueur de négation pa 'pas', lexicalisant la position NEG sur $\mathrm{S}$, et le cas de $k i$ 'qui' complémenteur, ayant en français les traits $+\mathrm{T}$, + nominatif. Dans le premier cas, on peut suggérer que $p a$ relexifie la particule de négation des langues d'Afrique de l'Ouest et occupe, dans AUX, la même position que cette dernière. Le deuxième cas est plus problématique. En haïtien, $k i$ est obligatoire quand le sujet est questionné, clivé ou relativisé (voir Koopman 1982 a et b), tel qu'illustré en (2), (3) et (4) respectivement.

(2) ki - num $k i$ te vini $\tilde{a}$

wh-personne qui TNS venir DET

'qui était venu'

(3) se ki-mun

c'est qui

$k i$ te vini $\tilde{a}$

qui TNS venir DET

C'est qui qui est venu

(4) mun nã

$k i$ te vini $\tilde{\mathrm{a}}$

personne DET qui TNS venir DET

L'homme qui est venu

$K i$ a-t-il été emprunté au français avec les traits qu'il a dans la langue source, c'est-à-dire comme complémenteur nominatif ou a-t-il été emprunté pour relexifier une position syntaxique autre dans la langue cible? Notre analyse comparative des structures cruciales des langues d'Afrique de l'Ouest, présumées avoir contribué à la formation de l'haïtien et des structures correspondantes en haïtien, (v.s. structures impliquant l'extraction du sujet) n'est pas assez avancée pour qu'il soit possible de 
fournir une réponse bien documentée à cette question. Nous laissons donc ce cas-problème en suspens pour l'instant.

L'analyse préliminaire de la formation des éléments lexicaux mineurs en haïtien implique donc deux processus. Le premier, emprunt d'items lexicaux majeurs du français réanalysés comme éléments lexicaux mineurs à l'intérieur de la langue cible pour lexicaliser les positions morphosyntaxiques déjà existantes dans la structure sous-jacente des langues d'Afrique de l'Ouest. Le second, la formation de mots, à partir de matériel lexical français, suivant les règles de formation de mots équivalents dans les langues d'Afrique de l'Ouest (v.g. les mots Wh). Mis à part le cas non encore analysé de $k i$, on n'observe en haïtien aucun cas d'emprunt au français d'items lexicaux mineurs.

\subsection{Discussion}

À l'aide des données analysées dans cette section, nous sommes en mesure d'offrir certaines réponses aux questions posées dans la section 2.2. Il est possible d'emprunter des items lexicaux de catégorie majeure et mineure (v.g. l'emprunt du si de l'espagnol en quechua). Les emprunts d'items lexicaux majeurs peuvent impliquer divers processus : lexification, relexification et translexification (e.g. le cas de la media lengua). Les emprunts d'items lexicaux mineurs ne peuvent impliquer que les processus de lexicalisation (v.g. emprunt du si de l'espagnol en quechua) et possiblement de relexification, mais pas le processus de translexification. La réanalyse interne s'avère être le seul mécanisme de création de position syntaxique (v.g. le cas du validateur -cus réanalysé comme complémenteur initial en quechua de Cochabamba), et le mécanisme le plus productif de création d'items lexicaux mineurs (v.g. le cas de la formation du lexique de l'haitien). Les items lexicaux empruntés doivent être insérés dans une catégorie syntaxique déjà existante dans la langue de sorte qu'une catégorie syntaxique ne peut être créée par l'emprunt (e.g. l'emprunt du si de l'espagnol en quechua, l'absence d'emprunt de prépositions à l'espagnol dans la media lengua). La discussion sur l'emprunt de conjonctions montre que c'est la syntaxe de la phrase qui constitue le niveau d'expression des contraintes sur l'emprunt lexical, la syntaxe du discours étant plus perméable à l'emprunt. Enfin nous avons vu qu'un élément emprunté peut s'insérer dans un paradigme déjà structuré (e.g. le cas de si emprunté comme mot Wh et inséré dans le paradigme des mots Wh en quechua de 
Cochabamba). Des recherches ultérieures devront documenter ce dernier point plus en détail.

Ces constatations peuvent être vues comme des contraintes sur l'emprunt lexical. Pour tous les cas considérés, et présumément pour les autres cas d'emprunt lexical, ces contraintes sont imposées par la structure de la langue cible et sont indépendantes de la structure de la langue source, ce qui restreint les changements linguistiques qui peuvent être causés par l'emprunt.

Regardons maintenant ces faits à la lumière de la théorie linguistique. Étant donné une théorie de la base dans laquelle les règles de base et le lexique sont intimement liés, les contraintes sur l'emprunt lexical se présentent comme une conséquence de la théorie de la base. Elles sont en fait prédictibles par la théorie. Il n'est donc pas nécessaire de les exprimer dans une grammaire, ces dernières découlant d'une théorie de la grammaire universelle. Les faits discutés sont également compatibles avec une théorie du changement qui limite le processus de changement linguistique à la réanalyse interne (e.g. Lightfoot 1979) et exclut les facteurs externes comme cause de changement linguistique.

\section{L'emprunt morphologique}

\subsection{Revue de la littérature}

La littérature sur l'emprunt contient des commentaires très variés sur les possibilités d'emprunt morphologique. Whitney (1881) écrit : «Whatever is more formal or structural in character remains to that degree free from the intrusion of foreign material.» Sapir (1927) affirme : «Nowhere do we find any but superficiel morphological inter influencing.» Dans Schuchardt (1928), on trouve le commentaire suivant : «Even closely knit structures like inflectional endings are not secure against invasion by foreign material.» Weinreich (1953) écrit : «The transfert of morphemes which are as strongly bound as inflectional endings in many European languages seems to be extremely rare.» De ces assertions, il ressort que les morphèmes flexionnels sont plus difficilement transférables d'une langue à une autre que les morphèmes qui ne jouent pas un rôle syntaxique. Cependant, dans la littérature, on trouve plusieurs cas de morphèmes dont on peut retracer l'origine dans une autre langue que la langue cible, autant des morphèmes flexionnels que des morphèmes dérivationnels. 


\subsection{Questions de recherche}

La théorie de la base, définie par Jackendoff (1977) et Chomsky (1981) fournit un cadre pour la formulation des questions de recherche sur l'emprunt morphologique. Selon cette théorie, les mots sont formés dans le lexique par des règles de formation de mots du type suggéré par Aronoff (1976) et Jackendoff (1977), puis insérés déjà formés dans des nœuds syntaxiques au moment de l'insertion lexicale.

Dans cette optique, 1) comment la morphologie est-elle empruntée puisque certains morphèmes dérivationnels et flexionnels ont une origine identifiable à une langue source différente de la langue cible? 2) quels sont les processus impliqués dans l'emprunt morphologique : relexification, réanalyse interne, etc.? 3) y a-t-il une différence entre la morphologie dérivationnelle et flexionnelle relativement à l'emprunt? Nous examinerons quelques cas d'emprunt morphologique qui nous permettent de discuter ces questions.

\section{3 Étude de cas}

\subsubsection{L'emprunt du morphème du pluriel de l'espagnol en quechua de Cochabamba}

Dans Lastra (1968) on trouve des noms quechuas marqués pour le pluriel au moyen du suffixe $-s$ de l'espagnol, au lieu du suffixe quechua équivalent : -kuna. J'aimerais suggérer que le $-s$ de l'espagnol n'a pas été emprunté directement mais qu'il a été emprunté comme partie intégrante des mots espagnols pluriels dont la forme pluriel a été empruntée comme forme non marquée. Dans un deuxième temps $-s$ aura été réanalysé par les bilingues comme marque du pluriel, puis inséré dans le paradigme des marqueurs de pluriel pour être utilisé avec des mots quechuas.

Les arguments qui montrent que $-s$ n'a pu être emprunté directement sont les suivants :

- premièrement, dans le dialecte de Cuzco, qui est plus conservateur que celui de Cochabamba et où l'on ne trouve pas $-s$ comme marqueur de pluriel, on trouve cependant une série d'emprunts à l'espagnol dans leur forme pluriel avec un sens singulier. Quand ces mots ont un sens pluriel, ils portent en plus la marque du pluriel -kuna telle qu'illustré en (1) : 


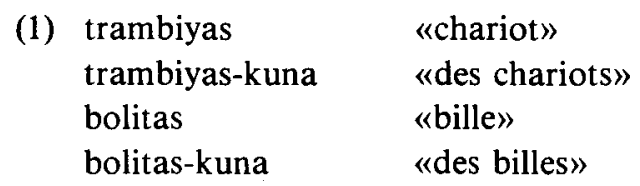

Dans ce cas le $-s$ n'est pas analysable comme marque du pluriel; il est partie intégrante du mot espagnol emprunté. Je suggère que cette phase, qui n'est plus observable à Cochabamba, a dû précéder la phase actuelle.

- deuxièmement, si - $s$ avait été emprunté directement à l'espagnol comme marque du pluriel, on s'attendrait à le trouver dans la même position dans le mot que le morphème de pluriel -kuna. Ainsi, on s'attendrait à trouver la forme (2b) équivalente à (2a). (2b) est cependant agrammaticale.

(2) a. bolita - yki - kuna

bille 2 PL

'tes billes'

b. *bolita - yki - s

bille 2 PL

'tes billes'

Dans un deuxième temps, $-s$ aura été identifié comme marqueur de pluriel par les bilingues. Pour les mots espagnols, il alterne alors avec -kuna, tel qu'illustré en (3).

(3) profesor-es

profesor-kuna 'les professeurs'

auto-s

auto-kuna 'les autos'

(Lastra 1968)

$-s$ et -kuna forment, à ce stade, un nouveau paradigme. Dans un troisième temps, $-s$ commence à être utilisé avec des mots quechuas en alternance avec $-k u n a$.

(4)

$$
\begin{aligned}
& \text { runa-kuna } \\
& \text { runa-s }
\end{aligned}
$$

'des gens'

'des gens'

Selon Lastra (1968), pour plusieurs locuteurs, $-s$ et -kuna apparaissent maintenant en distribution complémentaire : $-s$ dans l'environnement d'une voyelle, et -kuna dans l'environnement d'une consonne.

runa-s

'des gens' 
(6)

$$
\begin{array}{ll}
\text { supay-kuna } & \text { 'des diables' } \\
\text { *supay-ni-s } & \text { 'des diables' }
\end{array}
$$

Ma réanalyse des données de Cochabamba, présentées en vrac par Lastra (1968), suggère que -s n'a pas été emprunté directement mais comme partie intégrante des mots pluriels empruntés à l'espagnol comme forme non marquée, puis réanalysé comme marqueur de pluriel par un processus de changement interne pour ensuite être utilisé comme marque de pluriel avec les mots quechuas. Un autre changement interne subséquent à l'introduction de $-s$ dans le paradigme de morphème du pluriel a trait à la position du morphème du pluriel dans le mot. Dans le dialecte de Cuzco, et présumément dans le dialecte de Cochabamba également avant ce changement, le morphème du pluriel du nom vient après les marques de personnes, tel qu'illustré en (7).

(7) radical - personne - nombre

yan - ni - yki - kuna

'tes routes'

Après l'introduction du $-s$ de l'espagnol dans le paradigme des marqueurs de pluriel, l'ordre des morphèmes se trouve changé, tel qu'illustré en (8).

(8) a. bolita - s - ni - yki

bille PL (vide) $\mathrm{Z}$

'tes billes'

b. yan - kuna - yki

Faute PL Z

'tes routes'

Pour que ce changement dans l'ordre des suffixes puisse se produire, il fallait que $-s$ ait déjà été réanalysé comme morphème de pluriel créant ainsi une nouvelle position pour les morphèmes du pluriel dans le mot. La confirmation de cette assertion nous est fournie par l'agrammaticalité de (9).

(9) *bolitas - kuna - yki

bille PL 2

'tes billes'

\subsubsection{L'emprunt du suffixe de l'espagnol en media lengua}

Muysken (1980) rapporte que le suffixe $-n d o$ ) $-n d u$ est le seul suffixe productif de l'espagnol qui ait été emprunté en media lengua. Le suffixe 
-ndu apparaît en alternance avec les suffixes de subordination $-k p i$ (obviatif) et -sha (proximatif), tel qu'illustré en (1) et (2).

(1) a.

OBVIATIF

ahi - bi toka - $n d u$ isti sabi - nga-ma - bish

PRO LOC play SUB this know 3FU IND IND

'if we play there this one will know'

'si nous jouons là, celui-là le saura'

(Muysken 1980)

b.

OBVIATIF

bwenu uyari - xu - $k p i$ - ga, graba-nga- bu, disku - da...

well hear PR SUB TO record NOM BEN record AC

'when they have heard it well, to record it, the record...'

'quand ils l'ont entendu bien, pour l'enregistrer, le disque...'

(2) a.

PROXIMATIF

el-kuna-ga asi nustru abla-ri - $\mathrm{k}-\mathrm{ta}-\mathrm{s}$ uye- $n d u$-ga aprendi-n

he PL TO thus we talk RE NOM AC hearSUB TO learn 3 'they learn it when they hear what we speak'

'ils l'apprennent quand ils entendent ce que nous parlons' b.

PROXIMATIF

ahi - mi ya awanta-sha sinta-ri - na-ku-n

here AF stand SUB live RE PL 3

'there they live together standing it'

'là, ils vivent ensemble, en le supportant'

(Muysken 1980)

L'analyse de la fréquence d'occurrence de - $n d u$ dans un corpus par rapport à sa fonction syntaxique montre que - $n d u$ est en train de se substituer à -sha plutôt qu'à $-k p i$.

(3)

$\begin{array}{lr}\text { proximatif }(=s h a) & 81 \\ \text { obviatif }(=k p i) & 5 \\ \text { indéterminé } & 2 \\ \text { total des }-n d u & 88\end{array}$

(d'après Muysken 1980)

Quel processus l'emprunt de ce morphème implique-t-il? Ce cas est bien différent de celui de l'introduction du $-s$ de l'espagnol dans le quechua de 
Cochabamba. En effet, on ne trouve pas de formes permettant d'analyser, dans un premier temps, -ndo comme partie intégrante du mot emprunté.

$$
\text { *uyendo - sha - ga }
$$

(Muysken, communication personnelle)

Muysken analyse l'emprunt de -ndo comme un cas de relexification, par lequel -ndo emprunte les traits de -sha (très marginalement - $k p i$ ). Un tel cas de relexification est probablement très rare, mais possible. Il est explicable par le fait que - $n d o$, en espagnol de la région où la media lengua est parlée, jouit d'une distribution syntaxique beaucoup plus large qu'en espagnol standard (voir Muysken 1981), ce qui explique son éligibilité à relexifier un morphème du quechua lui étant similaire en plusieurs points.

Les deux exemples ci-haut mentionnés montrent que l'emprunt morphologique peut se faire selon deux processus: l'emprunt morphologique indirect impliquant l'emprunt d'un morphème comme partie intégrante d'une classe de mots empruntés pouvant par la suite être réanalysé à l'intérieur de la langue emprunteuse comme un morphème séparé et deuxièmement le processus de relexification d'un morphème d'une langue par un morphème équivalent d'une autre langue. Ces mêmes processus président-ils à l'emprunt de morphèmes dérivationnels? Nous considérons un seul cas, celui de l'emprunt de l'article français dans le montagnais.

\subsubsection{L'emprunt de l'article français en montagnais.}

Drapeau (1980) rapporte l'emprunt en montagnais de l'article défini du français tel qu'illustré dans les exemples suivants.

$\begin{array}{ll}\text { le.hu.p } & \text { 'soupe' } \\ \text { té.ga.le.p } & \text { 'crêpe' } \\ \text { lé.si.ga.rèt } & \text { 'cigarette' } \\ \text { la.hyèt } & \text { 'assiette' }\end{array}$

Drapeau montre que l'article n'a pas été emprunté indépendamment mais qu'il a été emprunté comme partie intégrante du mot français emprunté. L'argument principal en faveur d'une telle analyse est que les mots empruntés avec l'article sont sujets aux mêmes dérivations que les mots d'origine montagnaise, tel qu'illustré en (2).

$$
\begin{aligned}
& \text { ne. té.ga.le.p.em } \\
& 1 \text { crêpe POSS } \\
& \text { 'ma crêpe' }
\end{aligned}
$$


Les emprunts incluant les formes de l'article lé, le ou té sont des emprunts qui datent et qui respectent les contraintes phonotactiques du montagnais. Il y a par contre une quantité d'emprunts avec lé qui sont d'origine plus récente et qui ne sont pas intégrés phonologiquement. D'un point de vue sémantique lé n'est pas un simple calque de l'article les en français. On voit difficilement comment le de lesimã 'ciment', de lešumă̌ 'assurance chômage', de lekãsey 'conseil de bande', de lekafe 'café' pourrait être apparenté au les du français. Tous ces mots ont un sens singulier en montagnais comme d'ailleurs tous les emprunts marqués pour lé. Sémantiquement, lé en montagnais, est complètement vide. Drapeau conclut que, «le montagnais a donc emprunté un morphème au français mais lui confère une fonction bien différente de celle qu'il a dans la langue . source». (p. 38). Cette fonction est celle de marquer les nouveaux emprunts comme mots d'origine étrangère.

Les données présentées par Drapeau montrent que lé n'a pas été emprunté directement. La présence d'une liste imposante d'emprunts anciens contenant une forme de l'article devait être nécessaire pour que l'une des formes de l'article, en occurrence lé, ait pu être réanalysée comme préfixe dérivationnel marquant les nouveaux emprunts comme mots d'origine étrangère. Ce cas semble donc confirmer l'hypothèse selon laquelle les morphèmes ne sont pas empruntés directement mais sont empruntés comme partie intégrante des mots empruntés; ils peuvent ensuite être réanalysés comme morphèmes séparés dans la langue cible. Étant donné ce processus d'emprunt, il est prédictible que les morphèmes empruntés n'aient pas nécessairement le même sens dans la langue source et dans la langue cible.

\subsection{Discussion}

Si on compare cet exemple d'emprunt morphologique dérivationnel et l'emprunt du $-s$ du pluriel en quechua de Cochabamba, on constate que l'emprunt de morphèmes dérivationnels et flexionnels implique les mêmes processus : dans un premier temps, les mots sont empruntés dans leur forme fléchie comme forme non marquée, dans un deuxième temps un élément de cette classe de mots peut être réanalysé comme morphème séparé par un processus de réanalyse interne. Ces faits suggèrent que seulement les mots peuvent être empruntés directement et non le matériel morphologique. Le cas de -ndo en media lengua est différent. Il montre que la relexification 
peut impliquer du matériel morphologique. Les conditions permettant ce type d'emprunt devront être documentées.

L'hypothèse forte présentée dans cette section exclut les cas d'emprunt morphologique direct (c'est-à-dire ceux qui ne proviennent pas d'un processus de réanalyse interne), à moins que ne soit impliquée la relexification.

\section{L'emprunt syntaxique}

\subsection{Revue de la littérature}

L'emprunt syntaxique a été traité comme un phénomène de convergence, défini par Weinreich (1953) dans les termes suivants : des éléments divergents d'une variété $V_{1}$ par rapport à $V_{2}$ qui ne peuvent être attribués à un changement interne et qui montrent des similarités par rapport à la langue en contact, sont le résultat de convergence linguistique. L'étude la mieux connue relatant un cas de convergence linguistique est ceile de Gumperz et Wilson (1971). Ces derniers tentent de démontrer que les langues kannada, marathi, hindi/urdu parlées à Kupwar en Inde, ont subi une resyntaxification par un processus de convergence syntaxique, due à leur contact prolongé. L'effet de la resyntaxification est que les trois langues à Kupwar partagent maintenant une même grammaire même si leurs lexiques respectifs ont été maintenus. À part cette étude, les cas les plus courants d'emprunt syntaxique rapportés dans la littérature portent sur l'emprunt d'une construction syntaxique particulière. Les constructions relatives sont le plus fréquemment citées à ce chapitre. Par exemple, Hale (1971) suggère que l'absence de relatives enchâssées dans certaines langues (e.g. le walbiri) constitue un trou grammatical qui peut être comblé par l'emprunt d'une telle structure à une langue de contact qui la possède. Similairement, Karttunen et Lockhart (1976) rapportent que, alors qu'en nawatl classique on ne trouve pas de relatives enchâssées, la langue moderne a développé une telle construction correspondant à la structure des relatives en espagnol. Nadkarni (1975) tente de montrer que le konkani langue indoaryenne aurait emprunté au kannada, langue dravidienne, une construction relative avec mots Wh. Il considère ce cas comme «a very clear instance of structural borrowing at the level of syntax». (p. 672) et conclut que "what is remarkable about the syntax of Konkani relative clauses however is that it clearly demonstrates how linguistic convergence comes about under conditions of bilingualism and shows that. (...) even the deepest part of 
language structure is not impervious to radical changes». (p. 674).

À la lumière de ces analyses, dont on pourrait étendre la liste, on serait amené à conclure que les langues peuvent tout emprunter même des structures syntaxiques. L'implication d'un tel état de fait serait que des changements syntaxiques majeurs peuvent avoir une source externe. Si l'on se place dans l'optique d'une théorie de la grammaire du type de celle proposée dans l'introduction, on est forcé de remettre en question la pertinence de l'emprunt syntaxique dans le sens cité plus haut. N'y aurait-il pas une autre explication aux changements syntaxiques observés que celle de l'emprunt?

\subsection{Questions de recherche}

Des questions spécifiques peuvent être formulées en regard de l'emprunt syntaxique présumé, dans le cadre d'une théorie de la grammaire :

1) La notion de paramètre : dans quelle mesure les paramètres de la grammaire universelle contraignent-ils l'emprunt syntaxique? Comment un nouveau paramètre est-il introduit dans une langue?

2) La relation de gouvernement : cette relation joue-t-elle un rôle contraignant sur l'emprunt syntaxique?

3) La notion de noyau et périphérie : est-ce que les données d'emprunt appartiennent à la grammaire noyau ou à la périphérie, tel que suggéré par Chomsky (1981)? Quels aspects des phénomènes d'emprunt peuvent être expliqués dans le cadre d'une théorie adéquate de la grammaire et quels aspects ne le peuvent pas?

4) Le changement syntaxique : il a été proposé que le changement syntaxique ne peut être qu'interne (e.g. Lightfoot 1979). Comment expliquer les cas présumés d'emprunt syntaxique cités plus haut? N'y aurait-il pas une autre explication aux changements syntaxiques observés que celle de l'emprunt? Ces cas constituent-ils un contreexemple à la théorie que le changement syntaxique ne peut être qu'interne?

Je me limiterai à la discussion d'un seul cas d'emprunt syntaxique présumé, celui de l'emprunt présumé de la structure des relatives enchâssées de l'espagnol en quechua de Cochabamba. 


\section{3 Étude de cas: les relatives en quechua de Cochabamba}

En quechua de Cochabamba, le développement récent des relatives enchâssées tensées, introduites par des mots $\mathrm{Wh}$, construites sur le modèle de l'espagnol, ressemble étrangement aux cas d'emprunt syntaxique cités plus haut. On pourrait conclure à un cas d'emprunt syntaxique. Nous verrons cependant que tel n'est pas le cas. Examinons ces données en détail en les comparant avec des constructions similaires dans un dialecte plus conservateur du quechua, celui de Cuzco.

Bills et al. (1972, p. 273) consacrent un chapitre de leur livre à la formation des relatives (et questions indirectes) tensées enchâssées introduites par un mot Wh.

(1) rik $\mathrm{h}_{\mathrm{u}-\mathrm{ni}}$ warmi - ta [pi- ta- $\mathrm{cu}_{\mathrm{s}}$ felipe mǔc 'a-rqa]RELATIVE voir 1 femme $A C$ qui AC IND Philippe embrasser PA J'ai vu la femme que Philippe a embrassée

(2) tiyaku-n cay aqta- pi[may- man- cus ri- su- ncis] RELATIVE rester 3 cette ville LO où vers IND aller FU1 pers.pl. Il vit dans cette ville vers laquelle nous allons

$\begin{array}{lr}\text { ma yaca- ni-cu [ima- ta - cus ruwa- sag] } & \text { QUESTION } \\ \text { PAS savoir } 1 \text { NEG quoi AC IND faire 1FU } & \text { INDIRECTE }\end{array}$

Je ne sais pas quoi faire

Dans ces exemples, les mots Wh correspondent au paradigme des mots Wh des questions directes. Ils portent obligatoirement le suffixe $-\check{c} u s$, analysé dans la section 2 comme la réalisation morphologique d'un COMP initial. Les mots Wh portent le cas qui correspond à leur fonction dans la phrase relative. Dans ces phrases, le verbe contient un suffixe de temps fini. Ces phrases sont construites sur le modèle des relatives de l'espagnol. Ceci représente une innovation en quechua de Cochabamba, qu'on ne trouve pas dans les autres dialectes plus conservateurs du Quechua. (v.g. Cuzco).

Dans tous les dialectes quechuas, il existe deux façons générales de former des relatives. Les relatives enchâssées sont des phrases nominalisées dont la tête est un verbe nominalisé marqué pour un cas; ces constructions ne contiennent pas de pronom relatif. (Voir Lefebvre et Muysken (1982) et (à paraître)).

(4) [hamu- sha- q runa] ñaña- $\mathrm{y}$ - pa wasi - $\mathrm{n}$ - ta ri- $\mathrm{n}$ venir PR NOM homme sœur 1 GEN maison 3 AC aller 3 L'homme qui vient s'en va à la maison de ma soeur 
La fille que j'ai vu viendra

Les relatives adjointes à $S$ sont construites avec un verbe tensé et un complémenteur lexical final marqué pour le cas (Voir Lefebvre 1980, et Lefebvre et Muysken 1982).

(6) warmi hamu- sha- $\mathrm{n}$ cay - $\emptyset$, pay - mi rima-nqa femme venir PR 3 COMP NOM elle VAL parler 3FU La femme qui vient parlera

L'innovation observée en quechua de Cochabamba est-elle le résultat d'un emprunt syntaxique à l'espagnol? Nous montrerons que non et que, encore une fois, ce changement est attribuable à un développement interne. Les arguments en faveur d'une telle analyse sont les suivants. Premièrement, les relatives tensées construites avec des mots Wh existaient déjà en quechua au XVI ${ }^{\mathrm{e}}$ siècle tel qu'attesté par les données tirées du Huarochiri (1563). Les phrases (7) et (8) sont des relatives libres.

$$
\begin{aligned}
& \text { [may- pi - n t'anta ka- sqa], cay- man ri-rqa- nku } \\
& \text { où LO AF réunion être SD là vers aller PA 3PL }
\end{aligned}
$$

Ils vont là où est la réunion

(Huarochiri 1563)

(8) imayna - n kawsa- nki, chayshina wañu- nki comment AF vivre 2 ainsi mourir 2

Tu mourras comme tu a vécu

(Huarochiri 1563)

Ces relatives ne sont pas enchâssées mais adjointes à $\mathrm{S}$.

Deuxièmement, les relatives tensées avec mots Wh existent dans d'autres dialectes quechuas plus conservateurs que le dialecte de Cochabamba dont le dialecte de Cuzco, tel qu'illustré en (9).

$$
\begin{aligned}
& \text { riku- sha- ni warmi - ta, [pi- n hamu- n] } \\
& \text { voir PR } 1 \text { femme AC Wh VAL venir } 3 \\
& \text { Je vois la femme qui vient }
\end{aligned}
$$

Dans ce cas, les relatives ne sont pas enchâssées non plus mais coordonnées à S', tel qu'argumenté dans Lefebvre et Muysken (1982).

Ce qui semble constituer l'innovation à Cochabamba, c'est la possiblité d'enchâsser les relatives et les questions indirectes construites avec un mot 
Wh et un verbe à temps fini, la structure interne existant dans d'autres dialectes mais sans possibilité d'enchâssement (adjointes à $S$ ou coordonnées à la principale). Je suggère que c'est le développement d'un COMP initial, discuté dans la section 2 de cet article, qui a permis l'enchâssement des relatives et questions indirectes dans le quechua de Cochabamba. En effet, en quechua de Cochabamba, les mots Wh introduisant les relatives et les questions indirectes portent le suffixe -cus (suffixe validationnel réanalysé comme la réalisation morphologique d'un COMP initial); ils occupent une position initiale COMP (cf. (1), (2) et (3)). Dans l'exemple (9) tiré du dialecte de Cuzco, le mot Wh porte le validateur. Aucun argument syntaxique n'appuie une analyse selon laquelle le mot $\mathrm{Wh}$ occuperait une position Wh à l'extérieur de $S$ (pour une discussion sur la position du mot Wh dans ce cas voir Lefebvre et Muysken (à paraître)). Les phrases construites sur le modèle (9) n'ont donc pas de complémenteur et ne peuvent de ce fait être enchâssées. En quechua de Cochabamba, la réanalyse de -cus en complémenteur initial crée une position syntaxique de COMP initial dans laquelle les mots Wh peuvent être antéposés, ce qui permet l'enchâssement des relatives et des questions indirectes. Les faits du quechua de Cochabamba peuvent dont être expliqués de façon adéquate comme résultat d'un développement interne, sans qu'il $\mathbf{y}$ ait besoin de recours à l'emprunt syntaxique pour les expliquer.

À ce point, on peut se demander pourquoi les phrases subordonnées des langues qui ont un COMP final (comme le quechua ou le walbiri) ne peuvent être enchâssées quand elles ont une structure interne de verbe tensé et complémenteur lexical marqué pour le Cas. Je suggère que la réponse à cette question réside dans le fait que, dans ce cas, il n'y a pas d'opérateur dans COMP permettant à la règle de prédication d'opérer entre la tête et le nom relativisé dans la phrase enchâssée (pour une analyse de l'interprétation des relatives, voir Lefebvre et Muysken (à paraître)). Ce n'est que par un processus de développement interne d'un COMP initial permettant au mot Wh d'occuper une position COMP sur S', si non dans la syntaxe, dans la forme logique (voir Huang 1982), que les mots Wh peuvent servir d'opérateur et que l'interprétation relative peut prendre place.

\subsection{Discussion}

À la lumière des faits discutés plus haut, sur la base de l'analyse proposée, on peut faire l'hypothèse que l'emprunt syntaxique est virtuellement impossible. Pour qu'il se produise, il faut que la langue source 
contienne une structure qui soit compatible avec les changements internes possibles dans la langue cible. Ceci fait reposer l'emprunt éventuel sur la situation interne de la langue cible.

On peut présumer que si les cas d'emprunts syntaxiques postulés, dont ceux cités à la section 4.1 , étaient réanalysés à la lumière de l'analyse proposée pour le quechua, une explication similaire à celle du quechua pourrait rendre compte de leur développement.

\section{Conclusion}

Cet article avait pour but la formulation de questions de recherche pour l'étude des grammaires en contact dans le cadre de la grammaire générative :

1) Dans quel composant de la grammaire l'emprunt prend-il place? Les études de cas analysés dans cet article suggèrent que le composant lexical constitue le lieu privilégié de l'emprunt linguistique.

2) Quelles sont les contraintes sur l'emprunt linguistique? Dans tous les cas, l'emprunt est contraint par la structure de la langue cible. L'emprunt lexical doit respecter les catégories syntaxiques de la langue cible. L'emprunt syntaxique est considéré comme virtuellement impossible; il a été suggéré que pour qu'il se produise, il faut que la langue source contienne une structure qui soit compatible avec les changements internes possibles dans la langue cible. L'emprunt repose donc sur la situation interne de la langue cible. Aussi paradoxal que cela puisse sembler à première vue, la conclusion de cet article est que «l'on n'emprunte pas ce qu'on n'a pas».

3) Dans quelle mesure les processus d'emprunt (relexification, lexification, translexification) sont-ils contraints? Il s'avère que l'emprunt d'items lexicaux mineurs ne peut impliquer que les processus de lexification ou de relexification. L'emprunt de morphèmes dérivationnels et flexionnels se présente comme une conséquence d'un processus de réanalyse interne. L'emprunt direct de morphèmes liés ne peut se produire que s'il implique la relexification de morphèmes déjà existants dans la langue cible. Les analyses présentées prédisent que, dans ce cas, la translexification est exclue. Le processus de translexification ne peut donc impliquer que les items lexicaux majeurs sous réserve des contraintes exprimées plus haut. 
4) Une théorie auxiliaire est-elle nécessaire pour rendre compte des phénomènes d'emprunt linguistique? La théorie de la grammaire utilisée pour l'analyse des cas-types considérés dans cet article s'avère tout à fait adéquate pour rendre compte des phénomènes d'emprunt. En fait, il s'avère que les conclusions qui découlent des analyses proposées sont prédictibles par les théories du lexique de la base et des autres composants de la grammaire. Le recours à une théorie auxiliaire qui rendrait compte de l'emprunt s'avère donc superflue.

5) Quelle est la relation entre l'emprunt et le changement? Les analyses de cas-types proposées dans cet article mènent aux conclusions suivantes :

- Si tous les items lexicaux peuvent être empruntés, ces emprunts ne peuvent créer une catégorie syntaxique, ni introduire une construction syntaxique nouvelle dans la langue cible.

- L'emprunt d'items lexicaux mineurs ne peut introduire de traits syntaxiques dans la langue cible (ce qui va de pair avec le fait que ce type d'emprunt ne peut impliquer que la relexification).

- L'emprunt syntaxique étant virtuellement impossible, il ne peut être la cause de changement syntaxique dans une langue donnée.

- Le processus de réanalyse interne s'est avéré dans tous les cas étudiés comme le seul processus productif de changement.

Il ressort donc des analyses de cas que les changements causés par l'emprunt sont très limités (les types de changements possibles causés par l'emprunt restent cependant à être explorés). Ce fait découle également d'une théorie du changement linguistique selon laquelle le changement ne peut être qu'interne.

6) Ayant étudié les données de contact linguistique à partir d'une théorie de la grammaire, il est possible de faire la démarche inverse. Quelles conséquences ces résultats impliquent-ils pour une théorie de la grammaire?

Si l'emprunt est contraint par la structure interne de la langue cible et si les changements observés impliquant les éléments empruntés ne sont pas de nature distincte des changements observés autrement, il n'y a pas de raison pour exclure les données d'emprunt de l'ensemble des données utilisées dans l'argumentation linguistique comme c'était traditionnellement le cas (v.g. Chomsky 1965, 1981). À ma connaissance, il n'y a qu'en phonologie que les données d'emprunt ont 
été utilisées dans l'argumentation linguistique (v.g. Hymann, 1970 Kaye et Nykiel 1979). Les résultats des analyses présentées dans cet article suggèrent que les données d'emprunt lexical et morphologique peuvent également être utilisées dans l'argumentation morphologique et syntaxique. Par exemple, en morphologie, les données d'emprunt pourraient être utilisées dans le débat impliquant des théories opposées sur la structure du lexique. Les données présentées dans cet article semblent favoriser une approche lexicaliste où il n'existe pas de distinction entre la morphologie dérivationnelle et flexionnelle dans le processus de formation de mots (v.g. Williams 1981, Lefebvre et Muysken (à paraître, etc.) plutôt qu'une théorie qui distingue la morphologie dérivationnelle de la morphologie flexionnelle (v.g. Anderson 1982)). Au niveau syntaxique, les données d'emprunt pourraient être utilisées pour valider la notion de paramètre ou pour enrichir la discussion sur les notions de noyau et de périphérie dont le contenu est encore tellement obscur (v.g. VanRiemsdijk 1978, Chomsky 1979, Lefebvre et Muysken 1982).

Les données analysées plus haut peuvent également être utilisées dans le débat sur le changement linguistique.

Cet article se proposait d'ouvrir une nouvelle perspective de recherche pour l'étude des phénomènes d'emprunts. Il suscite cependant plusieurs questions. Les réponses aux questions de recherche proposées dans chacune des sections doivent être documentées plus à fond. De plus, les résultats des analyses présentées soulèvent une autre série de questions dont la discussion devra être alimentée par de nouvelles données. Par exemple, quelles sont les conditions (internes et/ou externes) qui rendent possible la relexification d'un morphème d'une langue par un morphème d'une autre langue? Qu'est-ce qui motive l'émergence d'un nouveau paramètre dans une langue et comment cela se produit-il? Quels sont les types de changements mineurs possibles qui peuvent être directement engendrés par l'emprunt?

Claire Lefebvre

Université du Québec à Montréal 


\section{Références}

ANDERSON, M. (1982), "Where is morphology», Linguistic Inquiry, 13, pp. 571-612. ARONOFF, M. (1976), «Word-Structure», thèse de Ph.D., M.I.T., Mass.

BILLS, G., R. TROIKE et A. VALLEJO (1972), An Introduction to Spoken Bolivian Quechua, Austin.

CHOMSKY, N. (1981), «Markedness and Core Grammar», dans A. Belletti et al., (eds), 1979 Proceedings of the Pisa GLOW Colloquium on Markedness, Scuola Superiore Normale, Pisa.

CHOMSKY, N. (1981), Lectures on Government and Binding, Dordrecht, Foris.

DRAPEAU, L. (1980), «Les emprunts au français en montagnais» dans Inuktitut et langues amérindiennes du Québec, Montréal, Les Presses de l'Université du Québec.

GUMPERZ, J. et R. WILSON (1971), «Convergence and Creolization : A case from the IndoAyran/Dravidian Border» dans D. Hymes (ed), Pidginization and Creolization of Languages, Cambridge University Press, pp. 151-169.

HALE, K. (1971), "The Adjoined Relative in Australian Languages», (ms).

HALE, K. (1975), «Gaps in Cultures and Grammars» dans M.D. Kindade, K. Hale, O. Werner, (eds), Linguistic and Anthropology in Honnor of CF Voegelin, Lisse, pp. 295-330.

HAUGEN, E. (1950), «The Analysis of Linguistic Borrowing», Language, $\mathrm{n}^{\circ} 26$, pp. 210-231 .

HUANG, J. (1982), «Between Syntax and Logical Form : A case Study in Chinese», Doctoral Dissertation, M.I.T.

HUAROCHIRI, Manuscrit en quechua, 1598-1607.

HUYBREGTS, R., H. VAN RIEMSDIJK (1982), Noam Chomsky on the Generative Enterprise, Dordrecht, Foris.

HYMAN, L. (1970) , «The Role of Borrowing in the Justification of Phonological Grammars», Studies in African Linguistics, vol. 1, pp. 1-48.

HYMES, D. (1971, Pidginization and Creolization of Languages, Cambridge University Press.

JACKENDOFF, R. (1977), $\bar{X}$ Syntax, Linguistic Inquiry Monograph, $\mathrm{n}^{\circ}$ 1, M.I.T. Press Cambridge, Mass.

KARTTUNEN, F. et J. LOCKHART (1976), Nawatl in the Middle Years, University of California Press, Berkeley.

KAYE, J. et B. Nikyel, «Loanwords and Asbract Phonotactic Constraints» dans RCL V1979, vol. $24, \mathrm{n}^{\circ} 2$, pp. 71-95.

KAYE, J. et C. LEFEBVRE (en préparation) "L'haitien : langues africaines relexifiées?"

KOOPMAN, H. (1982), «Les questions» dans C. Lefebvre et al., 1982, Syntaxe de l'haïtien, Ann Arbor, Karam Publications Inc.

KOOPMAN, H. (1982), "Les constructions relatives», dans C. Lefebvre et al., (1982).

KOOPMAN, H. (1982), "Control from COMP and comparative syntax» dans J. Kaye et al., (1982), Projet sur les langues $K R U$, Université du Québec à Montréal.

KOOPMAN, H. et C. LEFEBVRE (1982), «Pu: marqueur de mode, préposition et complémenteur", dans C. Lefebvre et al., (1982), Syntaxe de l'haitien, Ann Arbor, Karoma Press Inc. 
LASTRA, Y. (1968), Cochabamba Quechua syntax, The Hague, Mouton.

LEFÈBVRE, C. (1980), «Cases of lexical complementizers in Quechua and the theory of COMP», The journal of Linguistic Research, 1 (2), pp. 91-112.

LEFÈBVRE, C. (1982), «Introduction», dans C. Lefebvre et al., (réds.), Syntaxe de l'haitien, Ann Arbor, Karoma Publishers Inc.

LEFÈBVRE, C., (en préparation), «Relexification : the case of Haïtien creole».

LEFĖBVRE, C. et P. MUYSKEN (1982), «Relative clauses in Cuzco Quechua : interactions between core and periphery", Indiana University Linguistics Club.

LEFÈBVRE, C. et P. MUYSKEN (1978), «COMP in (Cuzco) Quechua», Cuny Forum Papers in Linguistics.

LEFÈBVRE, C. et P. MUYSKEN (1983), «Raising as move CASE», dans Linguistic Review $n^{\circ} 2$ : pp. 161-210.

LIGHTFOOT, D. (1979), Principles of diachronic Syntax, Cambridge University Press, London.

MEILLET, A. (1921), Linguistique historique et linguistique générale, La société de linguistique de Paris.

MUYSKEN, P. (1980), "Half-way between Spanish and Quechua. The case for relexification», dans A. Highfield et A. Valdman (eds.), Historicity and change in creole studies, Ann Arbor, Karoma Publishers Inc.

MUYSKEN, P. (1981), «The Spanish that Quechua Speakers learn : L learning as normfoverned behavior», Universiteit Van Amsterdam.

MUYSKEN, P. (1981), "Quechua word structure» dans F. Heny (ed.), Binding and Filtering, Longmans, London and MIT Press, Cambridge, Mass.

MUYSKEN, P. et C. LEFÈBVRE (à paraître), Nominalisations in Quechua (titre approximatif), Dordrecht, Reidll Publishing Company.

NADKARNI, M.V. (1975), "Bilingualism and syntactic change in Konkani» Language, vol. $51, \mathrm{n}^{\circ} 3$.

ROY, M.M. (1979), «Les conjonctions anglaises $B U T$ et $S O$ dans le français de Moncton», thèse de maîtrise, Université du Québec à Montréal.

SAPIR, E. (1927), Language, New York.

SANKOFF, G. (1980), "Variability and expansion in language and culture : cliticization in New Guinea tok Pisin» dans G. Sankoff (ed.), The Social life of language, University of Pennsylvania Press.

SANKOFF, G. et S. LABERGE (1973), «On the acquisition of native speakers by a language» dans Kivung, ${ }^{\circ}$ 6, pp. 32-47.

SANKOFF, G. et P. BROWN (1976), «The origins of syntax in discourse : a case study of tok pisin relatives" dans Language, $\mathrm{n}^{\circ} 52$, pp. 631-666.

SCHUCHARDT, H. (1928), Hugo Schuchardt-Brevier, (ed. L. Spitzer), Halle.

SELKIRK, E. (1982), The syntax of words, Linguistic Inquity monograph, $\mathrm{n}^{\circ} 7$, MIT Press, Cambridge, Mass.

SINGH, R. (1981), «Aspects of Language borrowing, English Leans in Hindi», Cambridge (Mass).

SWADESH, M. (1952), «Lexico-statistic Dating of Prehistoric Ethnic Contacts, with special reference to North American Indians and Eskimos" Proceedings of the Americain Philosophical Society, $\mathrm{n}^{\circ}$ 96, pp. 452-463. 
TESNIÈRE, L. (1939), Phonologie et mélange des langues, travaux du Cercle Linguistique de Prague, 8., pp. 83-93.

VAN RIEMSDIJK, H. (1978), On the binding nature of Prepositional phrases, Dordrecht, Foris.

WEINREICH, U. (1953), Languages in contact, Publications of the linguistic circle of New York, $\mathrm{n}^{\circ} 1$, New York.

WHITNEY, W.D. (1881), «On mixture is Language», Transaction of the American Philosophical Association, $\mathrm{n}^{\circ} 12$, pp. 1-26.

WILLIAMS, E. (1981), «On the notions» lexically related «an» head of a word». Linguistic Inquity. vol. $12 . \mathrm{n}^{\circ} 2$, pp. 245-275. 